Network Working Group

Request for Comments: 3023

Obsoletes: 2376

Updates: 2048

Category: Standards Track
M. Murata

IBM Tokyo Research Laboratory

S. St.Laurent simonstl.com

D. Kohn

Skymoon Ventures January 2001

XML Media Types

Status of this Memo

This document specifies an Internet standards track protocol for the Internet community, and requests discussion and suggestions for improvements. Please refer to the current edition of the "Internet Official Protocol Standards" (STD 1) for the standardization state and status of this protocol. Distribution of this memo is unlimited.

Copyright Notice

Copyright (C) The Internet Society (2001). All Rights Reserved.

Abstract

This document standardizes five new media types -- text/xml, application/xml, text/xml-external-parsed-entity, application/xmlexternal-parsed-entity, and application/xml-dtd -- for use in exchanging network entities that are related to the Extensible Markup Language (XML). This document also standardizes a convention (using the suffix '+xml') for naming media types outside of these five types when those media types represent XML MIME (Multipurpose Internet Mail Extensions) entities. XML MIME entities are currently exchanged via the HyperText Transfer Protocol on the World Wide Web, are an integral part of the WebDAV protocol for remote web authoring, and are expected to have utility in many domains.

Major differences from RFC 2376 are (1) the addition of text/xmlexternal-parsed-entity, application/xml-external-parsed-entity, and application/xml-dtd, (2) the '+xml' suffix convention (which also updates the RFC 2048 registration process), and (3) the discussion of "utf-16le" and "utf-16be". 
Table of Contents

1. Introduction . . . . . . . . . . . . . . . . . . . . . 3

2. Notational Conventions . . . . . . . . . . . . . . . . . . . 4

3. XML Media Types . . . . . . . . . . . . . . . . . . . . 5

3.1 Text/xml Registration . . . . . . . . . . . . . . . . 7

3.2 Application/xml Registration . . . . . . . . . . . . . . . . 9

3.3 Text/xml-external-parsed-entity Registration . . . . . . . .11

3.4 Application/xml-external-parsed-entity Registration . • . 12

3.5 Application/xml-dtd Registration . . . . . . . . . . . . 13

3.6 Summary . . . . . . . . . . . . . . . . . . . . . . . 14

4. The Byte Order Mark (BOM) and Conversions to/from the UTF-16 Charset . . . . . . . . . . . . . . . . . . 15

5. Fragment Identifiers . . . . . . . . . . . . . . 15

6. The Base URI . . . . . . . . . . . . . . . . . . . . 15

7. A Naming Convention for XML-Based Media Types . . . . . 16

7.1 Referencing . • . . . . . . . . . . . . . . . . . . . . 18

8. Examples . . . . . . . . . . . . . . . . . . . . . 18

8.1 Text/xml with UTF-8 Charset . . . . . . . . . . . . . . . 19

8.2 Text/xml with UTF-16 Charset . . . . . . . . . . . . . . . . 19

8.3 Text/xml with UTF-16BE Charset. . . . . . . . . . . . . . 19

8.4 Text/xml with ISO-2022-KR Charset . . . . . . . . . . . 20

8.5 Text/xml with Omitted Charset . . . . . . . . . . . . 20

8.6 Application/xml with UTF-16 Charset . . . . . . . . . . . 20

8.7 Application/xml with UTF-16BE Charset . . . . . . . . . . . 21

8.8 Application/xml with ISO-2022-KR Charset . . . . . . . . . . 21

8.9 Application/xml with Omitted Charset and UTF-16 XML MIME

Entity . . . . . . . . . . . . . . . . . . . . . . 21

8.10 Application/xml with Omitted Charset and UTF-8 Entity • • 22

8.11 Application/xml with Omitted Charset and Internal Encoding Declaration . . . . . . . . . . . . . . . . . . 22

8.12 Text/xml-external-parsed-entity with UTF-8 Charset . . . . 22

8.13 Application/xml-external-parsed-entity with UTF-16 Charset . 23

8.14 Application/xml-external-parsed-entity with UTF-16BE Charset 23

8.15 Application/xml-dtd . . . . . . . . . . . . . . . . 23

8.16 Application/mathml+xml . . . . . . . . . . . . . . . . . 24

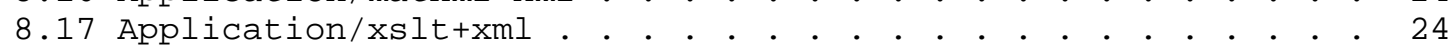

8.18 Application/rdftxml • . . . . . . . . . . . . . . . . . . . 24

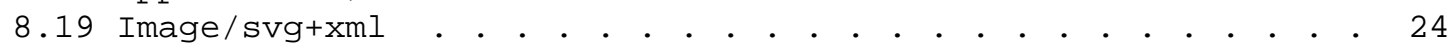

8.20 INCONSISTENT EXAMPLE: Text/xml with UTF-8 Charset . . . . 25

9. IANA Considerations . . . . . . . . . . . . . . . . 25

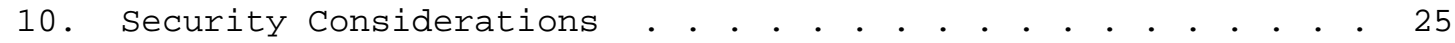
References . . . . . . . . . . . . . . . . . . 27 Authors' Addresses . . . . . . . . . . . . . . . . . 31

A. Why Use the 'txml' Suffix for XML-Based MIME Types? . . . . 32

A.1 Why not just use text/xml or application/xml and let the XML processor dispatch to the correct application based on the referenced DTD? 
A.2 Why not create a new subtree (e.g., image/xml.svg) to represent XML MIME types? . . . . . . . . . . . . . 32

A.3 Why not create a new top-level MIME type for XML-based media types? . . . . . . . . . . . . . . . . . . . . . . 32

A.4 Why not just have the MIME processor 'sniff' the content to determine whether it is XML? . . . . . . . . . . . . . 33

A.5 Why not use a MIME parameter to specify that a media type uses XML syntax? • • • • • • • • • • • • • • • • • • • • • 33

A. 6 How about labeling with parameters in the other direction (e.g., application/xml; Content-Feature=iotp) ? . . . . . . 34

A.7 How about a new superclass MIME parameter that is defined to apply to all MIME types (e.g., Content-Type: application/iotp; \$superclass=xml) ? $\quad$.

A.8 What about adding a new parameter to the Content-Disposition header or creating a new Content-Structure header to indicate XML syntax? . . . . . . . . . . . . . . . . 35

A.9 How about a new Alternative-Content-Type header? . . . . . . 35

A.10 How about using a conneg tag instead (e.g., accept-features: $(\operatorname{syntax}=x m l))$ ? . . . . . . . . . . . . . . . . . . . . . 35

A.11 How about a third-level content-type, such as text/xml/rdf? 35

A.12 Why use the plus ('+') character for the suffix '+xml'? • . 36

A.13 What is the semantic difference between application/foo and application/footxml? . . . . . . . . . . . . . . . . 36

A.14 What happens when an even better markup language (e.g., EBML) is defined, or a new category of data? . . . . . . . 36

A.15 Why must I use the '+xml' suffix for my new XML-based media type? . . . . . . . . . . . . . . . . . . . . . 37

B. Changes from RFC 2376 . . . . . . . . . . . . . . . . . 37

C. Acknowledgements . . . . . . . . . . . . . . . . . 38 Full Copyright Statement . . . . . . . . . . . . . . . 39

1. Introduction

The World Wide Web Consortium has issued Extensible Markup Language (XML) 1.0 (Second Edition) [XML]. To enable the exchange of XML network entities, this document standardizes five new media types -text/xml, application/xml, text/xml-external-parsed-entity, application/xml-external-parsed-entity, and application/xml-dtd -- as well as a naming convention for identifying XML-based MIME media types.

XML entities are currently exchanged on the World Wide Web, and XML is also used for property values and parameter marshalling by the WebDAV[RFC2518] protocol for remote web authoring. Thus, there is a need for a media type to properly label the exchange of XML network entities. 
Although XML is a subset of the Standard Generalized Markup Language (SGML) ISO 8879[SGML], which has been assigned the media types text/sgml and application/sgml, there are several reasons why use of text/sgml or application/sgml to label XML is inappropriate. First, there exist many applications that can process XML, but that cannot process SGML, due to SGML's larger feature set. Second, SGML applications cannot always process XML entities, because XML uses features of recent technical corrigenda to SGML. Third, the definition of text/sgml and application/sgml in [RFC1874] includes parameters for SGML bit combination transformation format (SGMLbctf), and SGML boot attribute (SGML-boot). Since XML does not use these parameters, it would be ambiguous if such parameters were given for an XML MIME entity. For these reasons, the best approach for labeling XML network entities is to provide new media types for XML.

Since XML is an integral part of the WebDAV Distributed Authoring Protocol, and since World Wide Web Consortium Recommendations have conventionally been assigned IETF tree media types, and since similar media types (HTML, SGML) have been assigned IETF tree media types, the XML media types also belong in the IETF media types tree.

Similarly, XML will be used as a foundation for other media types, including types in every branch of the IETF media types tree. To facilitate the processing of such types, media types based on XML, but that are not identified using text/xml or application/xml, SHOULD be named using a suffix of 'txml' as described in section 7. This will allow XML-based tools -- browsers, editors, search engines, and other processors -- to work with all XML-based media types.

\section{Notational Conventions}

The key words "MUST", "MUST NOT", "REQUIRED", "SHALL", "SHALL NOT", "SHOULD", "SHOULD NOT", "RECOMMENDED", "MAY", and "OPTIONAL" in this document are to be interpreted as described in [RFC2119].

As defined in [RFC2781], the three charsets "utf-16", "utf-16le", and "utf-16be" are used to label UTF-16 text. In this document, "the UTF-16 family" refers to those three charsets. By contrast, the phrases "utf-16" or UTF-16 in this document refer specifically to the single charset "utf-16".

As sometimes happens between two communities, both MIME and XML have defined the term entity, with different meanings. Section 2.4 of [RFC2045] says:

"The term 'entity' refers specifically to the MIME-defined header fields and contents of either a message or one of the parts in the body of a multipart entity." 
Section 4 of [XML] says:

"An XML document may consist of one or many storage units" called entities that "have content" and are normally "identified by name".

In this document, "XML MIME entity" is defined as the latter (an XML entity) encapsulated in the former (a MIME entity).

3. XML Media Types

This document standardizes five media types related to XML MIME entities: text/xml, application/xml, text/xml-external-parsed-entity, application/xml-external-parsed-entity, and application/xml-dtd. Registration information for these media types is described in the sections below.

Within the XML specification, XML MIME entities can be classified into four types. In the XML terminology, they are called "document entities", "external DTD subsets", "external parsed entities", and "external parameter entities". The media types text/xml and application/xml MAY be used for "document entities", while text/xmlexternal-parsed-entity or application/xml-external-parsed-entity SHOULD be used for "external parsed entities". The media type application/xml-dtd SHOULD be used for "external DTD subsets" or "external parameter entities". application/xml and text/xml MUST NOT be used for "external parameter entities" or "external DTD subsets", and MUST NOT be used for "external parsed entities" unless they are also well-formed "document entities" and are referenced as such. Note that [RFC2376] (which this document obsoletes) allowed such usage, although in practice it is likely to have been rare.

Neither external DTD subsets nor external parameter entities parse as XML documents, and while some XML document entities may be used as external parsed entities and vice versa, there are many cases where the two are not interchangeable. XML also has unparsed entities, internal parsed entities, and internal parameter entities, but they are not XML MIME entities.

If an XML document -- that is, the unprocessed, source XML document -- is readable by casual users, text/xml is preferable to application/xml. MIME user agents (and web user agents) that do not have explicit support for text/xml will treat it as text/plain, for example, by displaying the XML MIME entity as plain text. Application/xml is preferable when the XML MIME entity is unreadable by casual users. Similarly, text/xml-external-parsed-entity is 
preferable when an external parsed entity is readable by casual users, but application/xml-external-parsed-entity is preferable when a plain text display is inappropriate.

NOTE: Users are in general not used to text containing tags such as <price>, and often find such tags quite disorienting or annoying. If one is not sure, the conservative principle would suggest using application/* instead of text/* so as not to put information in front of users that they will quite likely not understand.

The top-level media type "text" has some restrictions on MIME entities and they are described in [RFC2045] and [RFC2046]. In particular, the UTF-16 family, UCS-4, and UTF-32 are not allowed (except over HTTP [RFC2616], which uses a MIME-like mechanism). Thus, if an XML document or external parsed entity is encoded in such character encoding schemes, it cannot be labeled as text/xml or text/xml-external-parsed-entity (except for HTTP).

Text/xml and application/xml behave differently when the charset parameter is not explicitly specified. If the default charset (i.e., US-ASCII) for text/xml is inconvenient for some reason (e.g., bad web servers), application/xml provides an alternative (see "Optional parameters" of application/xml registration in section 3.2). The same rules apply to the distinction between text/xml-externalparsed-entity and application/xml-external-parsed-entity.

XML provides a general framework for defining sequences of structured data. In some cases, it may be desirable to define new media types that use XML but define a specific application of XML, perhaps due to domain-specific security considerations or runtime information. Furthermore, such media types may allow UTF-8 or UTF-16 only and prohibit other charsets. This document does not prohibit such media types and in fact expects them to proliferate. However, developers of such media types are STRONGLY RECOMMENDED to use this document as a basis for their registration. In particular, the charset parameter SHOULD be used in the same manner, as described in section 7.1, in order to enhance interoperability.

An XML document labeled as text/xml or application/xml might contain namespace declarations, stylesheet-linking processing instructions (PIs), schema information, or other declarations that might be used to suggest how the document is to be processed. For example, a document might have the XHTML namespace and a reference to a CSS stylesheet. Such a document might be handled by applications that would use this information to dispatch the document for appropriate processing. 


\subsection{Text/xml Registration}

MIME media type name: text

MIME subtype name: xml

Mandatory parameters: none

Optional parameters: charset

Although listed as an optional parameter, the use of the charset parameter is STRONGLY RECOMMENDED, since this information can be used by XML processors to determine authoritatively the character encoding of the XML MIME entity. The charset parameter can also be used to provide protocol-specific operations, such as charsetbased content negotiation in HTTP. "utf-8" [RFC2279] is the recommended value, representing the UTF-8 charset. UTF-8 is supported by all conforming processors of [XML].

If the XML MIME entity is transmitted via HTTP, which uses a MIME-like mechanism that is exempt from the restrictions on the text top-level type (see section 19.4.1 of [RFC2616]), "utf-16" [RFC2781]) is also recommended. UTF-16 is supported by all conforming processors of [XML]. Since the handling of $\mathrm{CR}, \mathrm{LF}$ and NUL for text types in most MIME applications would cause undesired transformations of individual octets in UTF-16 multi-octet characters, gateways from HTTP to these MIME applications MUST transform the XML MIME entity from text/xml; charset="utf-16" to application/xml; charset="utf-16".

Conformant with [RFC2046], if a text/xml entity is received with the charset parameter omitted, MIME processors and XML processors MUST use the default charset value of "us-ascii"[ASCII]. In cases where the XML MIME entity is transmitted via HTTP, the default charset value is still "us-ascii". (Note: There is an inconsistency between this specification and HTTP/1.1, which uses ISO-8859-1[ISO8859] as the default for a historical reason. Since XML is a new format, a new default should be chosen for better I18N. US-ASCII was chosen, since it is the intersection of UTF-8 and ISO-8859-1 and since it is already used by MIME.)

There are several reasons that the charset parameter is authoritative. First, some MIME processing engines do transcoding of MIME bodies of the top-level media type "text" without reference to any of the internal content. Thus, it is possible that some agent might change text/xml; charset="iso-2022-jp" to text/xml; charset="utf-8" without modifying the encoding declaration of an XML document. Second, text/xml must be 
compatible with text/plain, since MIME agents that do not understand text/xml will fallback to handling it as text/plain. If the charset parameter for text/xml were not authoritative, such fallback would cause data corruption. Third, recent web servers have been improved so that users can specify the charset parameter. Fourth, [RFC2130] specifies that the recommended specification scheme is the "charset" parameter.

Since the charset parameter is authoritative, the charset is not always declared within an XML encoding declaration. Thus, special care is needed when the recipient strips the MIME header and provides persistent storage of the received XML MIME entity (e.g., in a file system). Unless the charset is UTF-8 or UTF-16, the recipient SHOULD also persistently store information about the charset, perhaps by embedding a correct XML encoding declaration within the XML MIME entity

Encoding considerations: This media type MAY be encoded as appropriate for the charset and the capabilities of the underlying MIME transport. For 7-bit transports, data in UTF-8 MUST be encoded in quoted-printable or base64. For 8-bit clean transport (e.g., 8BITMIME[RFC1652] ESMTP or NNTP[RFC0977]), UTF-8 does not need to be encoded. Over HTTP[RFC2616], no content-transferencoding is necessary and UTF-16 may also be used.

Security considerations: See Section 10 .

Interoperability considerations: XML has proven to be interoperable across WebDAV clients and servers, and for import and export from multiple XML authoring tools. For maximum interoperability, validating processors are recommended. Although non-validating processors may be more efficient, they are not required to handle all features of XML. For further information, see sub-section 2.9 "Standalone Document Declaration" and section 5 "Conformance" of [XML ].

Published specification: Extensible Markup Language (XML) 1.0 (Second Edition) [XML].

Applications which use this media type: XML is device-, platform-, and vendor-neutral and is supported by a wide range of Web user agents, WebDAV[RFC2518] clients and servers, as well as XML authoring tools.

Additional information: 


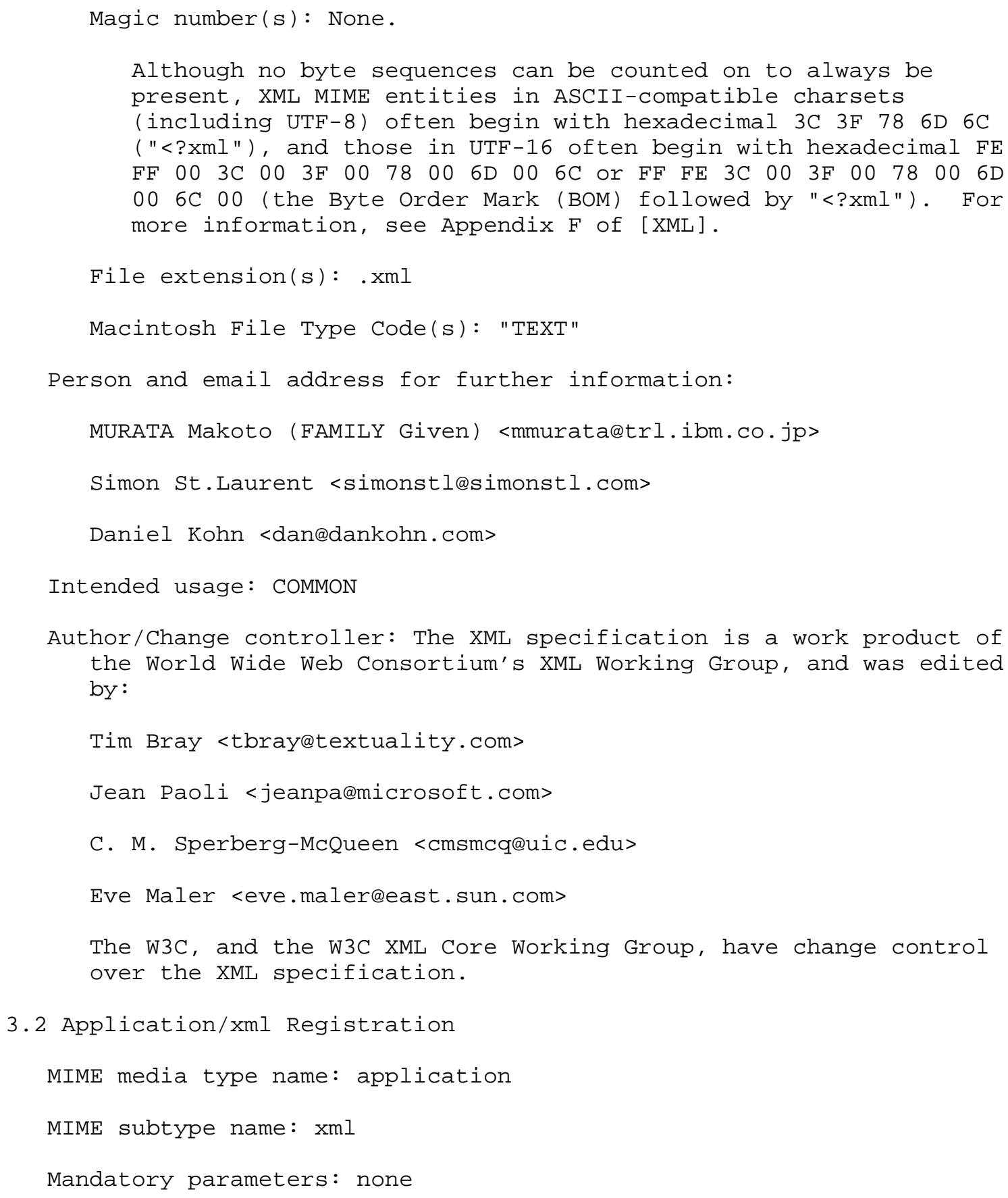


Optional parameters: charset

Although listed as an optional parameter, the use of the charset parameter is STRONGLY RECOMMENDED, since this information can be used by XML processors to determine authoritatively the charset of the XML MIME entity. The charset parameter can also be used to provide protocol-specific operations, such as charset-based content negotiation in HTTP.

"utf-8" [RFC2279] and "utf-16" [RFC2781] are the recommended values, representing the UTF-8 and UTF-16 charsets, respectively. These charsets are preferred since they are supported by all conforming processors of [XML].

If an application/xml entity is received where the charset parameter is omitted, no information is being provided about the charset by the MIME Content-Type header. Conforming XML processors MUST follow the requirements in section 4.3 .3 of [XML] that directly address this contingency. However, MIME processors that are not XML processors SHOULD NOT assume a default charset if the charset parameter is omitted from an application/xml entity.

There are several reasons that the charset parameter is authoritative. First, recent web servers have been improved so that users can specify the charset parameter. Second, [RFC2130] specifies that the recommended specification scheme is the "charset" parameter.

On the other hand, it has been argued that the charset parameter should be omitted and the mechanism described in Appendix $F$ of [XML] (which is non-normative) should be solely relied on. This approach would allow users to avoid configuration of the charset parameter; an XML document stored in a file is likely to contain a correct encoding declaration or BOM (if necessary), since the operating system does not typically provide charset information for files. If users would like to rely on the encoding declaration or BOM and to hide charset information from protocols, they may determine not to use the parameter.

Since the charset parameter is authoritative, the charset is not always declared within an XML encoding declaration. Thus, special care is needed when the recipient strips the MIME header and provides persistent storage of the received XML MIME entity (e.g., in a file system). Unless the charset is UTF-8 or UTF-16, the recipient SHOULD also persistently store information about the charset, perhaps by embedding a correct XML encoding declaration within the XML MIME entity 
Encoding considerations: This media type MAY be encoded as appropriate for the charset and the capabilities of the underlying MIME transport. For 7-bit transports, data in either UTF-8 or UTF-16 MUST be encoded in quoted-printable or base64. For 8-bit clean transport (e.g., 8BITMIME[RFC1652] ESMTP or NNTP[RFC0977]), UTF-8 is not encoded, but the UTF-16 family MUST be encoded in base64. For binary clean transports (e.g., HTTP [RFC2616]), no content-transfer-encoding is necessary.

Security considerations: See Section 10.

Interoperability considerations: Same as section 3.1.

Published specification: Same as section 3.1.

Applications which use this media type: Same as section 3.1.

Additional information: Same as Section 3.1.

Person and email address for further information: Same as Section 3.1 .

Intended usage: COMMON

Author/Change controller: Same as section 3.1.

3.3 Text/xml-external-parsed-entity Registration

MIME media type name: text

MIME subtype name: xml-external-parsed-entity

Mandatory parameters: none

Optional parameters: charset

The charset parameter of text/xml-external-parsed-entity is handled the same as that of text/xml as described in section 3.1 .

Encoding considerations: Same as Section 3.1.

Security considerations: See Section 10 .

Interoperability considerations: XML external parsed entities are as interoperable as XML documents, though they have a less tightly constrained structure and therefore need to be referenced by XML documents for proper handling by XML processors. Similarly, XML documents cannot be reliably used as external parsed entities 


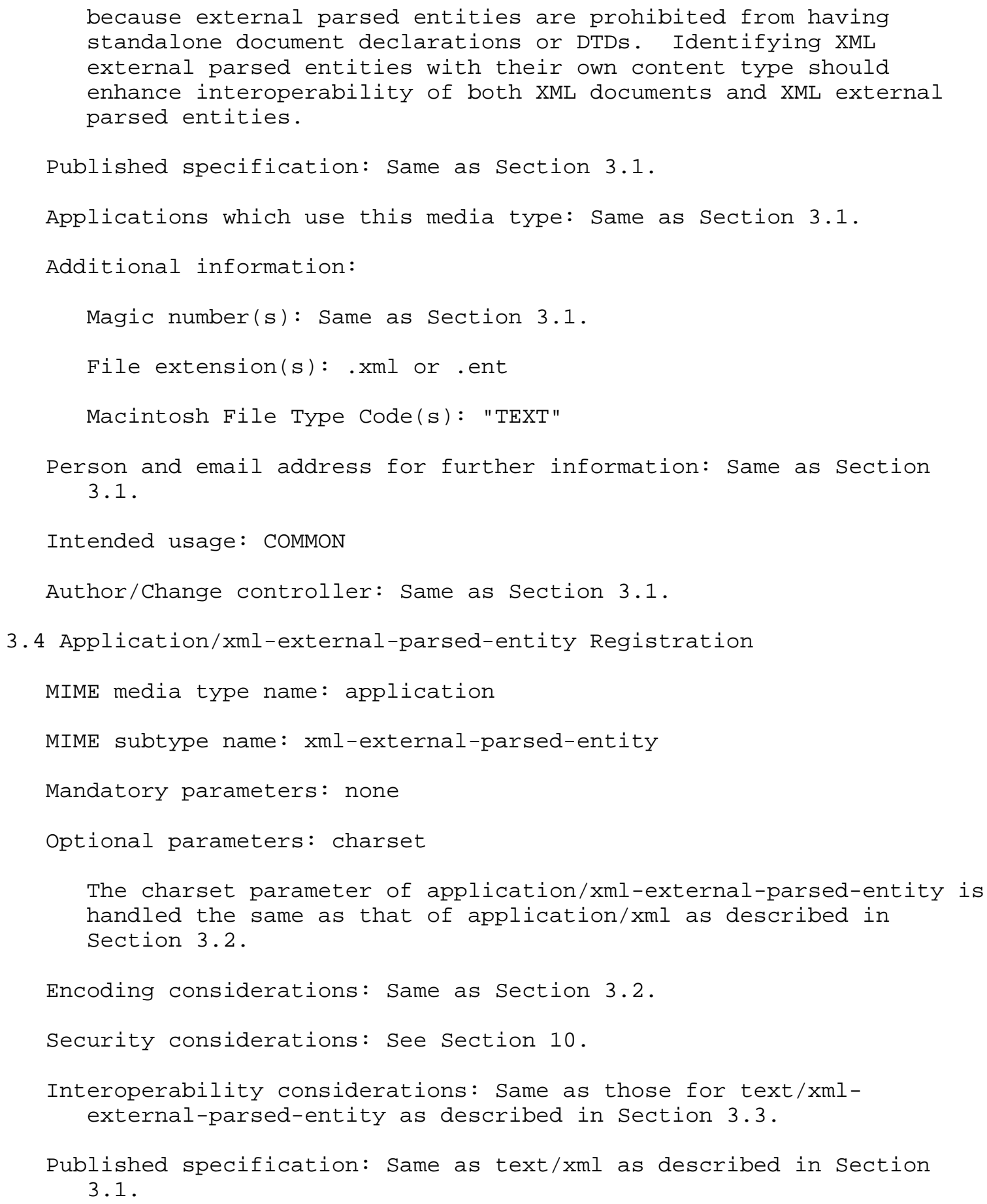




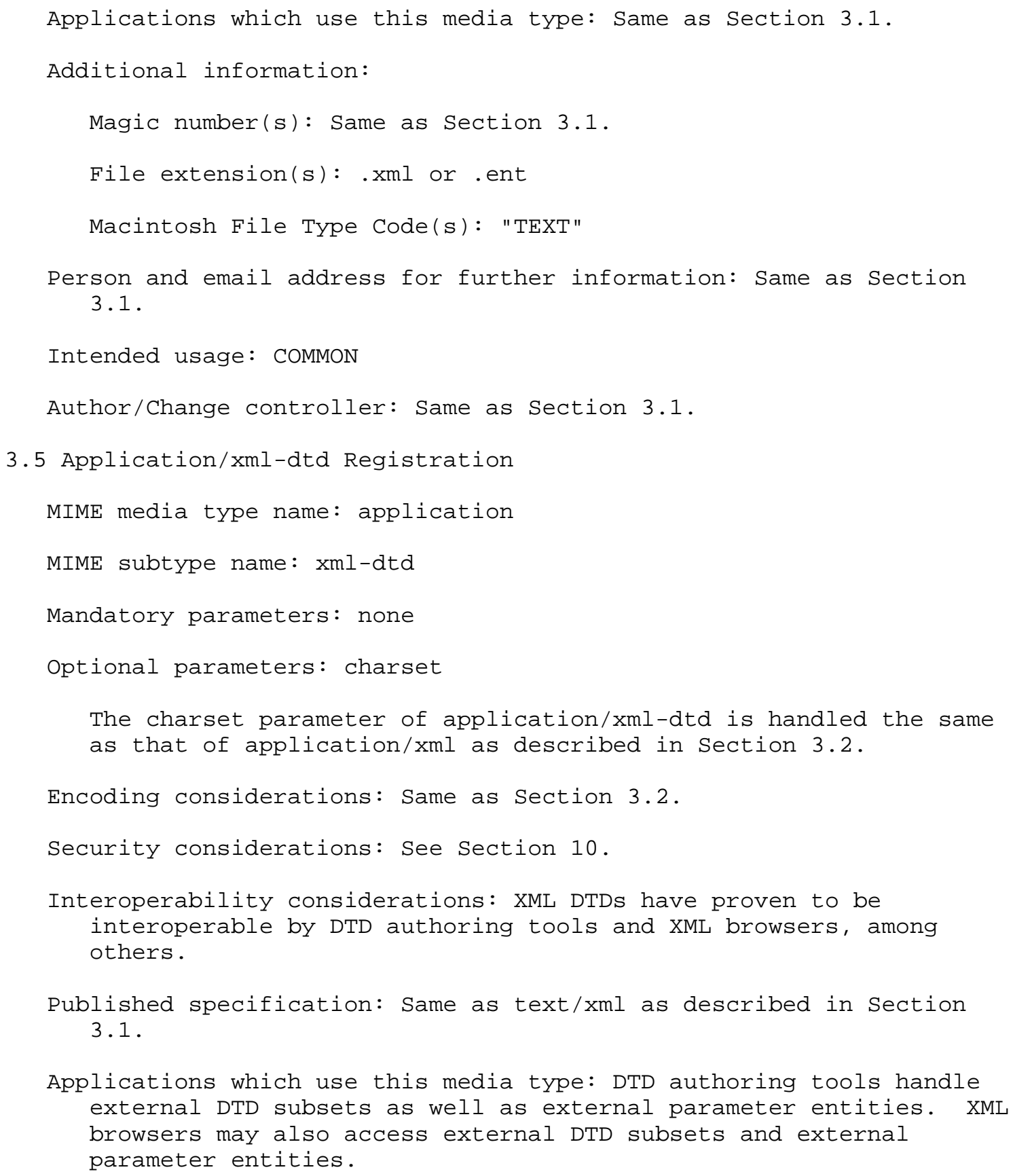


Additional information:

Magic number(s): Same as section 3.1.

File extension(s): .dtd or .mod

Macintosh File Type Code(s): "TEXT"

Person and email address for further information: Same as Section 3.1 .

Intended usage: COMMON

Author/Change controller: Same as Section 3.1.

3. 6 summary

The following list applies to text/xml, text/xml-external-parsedentity, and XML-based media types under the top-level type "text" that define the charset parameter according to this specification:

o Charset parameter is strongly recommended.

- If the charset parameter is not specified, the default is "usascii". The default of "iso-8859-1" in HTTP is explicitly overridden.

- No error handling provisions.

- An encoding declaration, if present, is irrelevant, but when saving a received resource as a file, the correct encoding declaration SHOULD be inserted.

The next list applies to application/xml, application/xml-externalparsed-entity, application/xml-dtd, and XML-based media types under top-level types other than "text" that define the charset parameter according to this specification:

- Charset parameter is strongly recommended, and if present, it takes precedence.

- If the charset parameter is omitted, conforming XML processors MUST follow the requirements in section 4.3 .3 of [XML]. 
4. The Byte Order Mark (BOM) and Conversions to/from the UTF-16 Charset

Section 4.3.3 of [XML] specifies that XML MIME entities in the charset "utf-16" MUST begin with a byte order mark (BOM), which is a hexadecimal octet sequence $0 x F E$ OxFF (or $0 x F F 0 x F E$, depending on endian). The XML Recommendation further states that the BOM is an encoding signature, and is not part of either the markup or the character data of the XML document.

Due to the presence of the BOM, applications that convert XML from "utf-16" to a non-Unicode encoding MUST strip the BOM before conversion. Similarly, when converting from another encoding into "utf-16", the BOM MUST be added after conversion is complete.

In addition to the charset "utf-16", [RFC2781] introduces "utf-16le" (little endian) and "utf-16be" (big endian) as well. The BOM is prohibited for these charsets. When an XML MIME entity is encoded in "utf-16le" or "utf-16be", it MUST NOT begin with the BOM but SHOULD contain an encoding declaration. Conversion from "utf-16" to "utf16be" or "utf-16le" and conversion in the other direction MUST strip or add the BOM, respectively.

5. Fragment Identifiers

Section 4.1 of [RFC2396] notes that the semantics of a fragment identifier (the part of a URI after a "\#") is a property of the data resulting from a retrieval action, and that the format and interpretation of fragment identifiers is dependent on the media type of the retrieval result.

As of today, no established specifications define identifiers for XML media types. However, a working draft published by W3C, namely "XML Pointer Language (XPointer)", attempts to define fragment identifiers for text/xml and application/xml. The current specification for XPointer is available at http://www.w3.org/TR/xptr.

6. The Base URI

Section 5.1 of [RFC2396] specifies that the semantics of a relative URI reference embedded in a MIME entity is dependent on the base URI. The base URI is either (1) the base URI embedded in the MIME entity, (2) the base URI of the encapsulating MIME entity, (3) the URI used to retrieve the MIME entity, or (4) the application-dependent default base URI, where (1) has the highest precedence. [RFC2396] further specifies that the mechanism for embedding the base URI is dependent on the media type. 
As of today, no established specifications define mechanisms for embedding the base URI in XML MIME entities. However, a Proposed Recommendation published by W3C, namely "XML Base", attempts to define such a mechanism for text/xml, application/xml, text/xmlexternal-parsed-entity, and application/xml-external-parsed-entity. The current specification for XML Base is available at http://www.w3.org/TR/xmlbase.

7. A Naming Convention for XML-Based Media Types

This document recommends the use of a naming convention (a suffix of ' +xml') for identifying XML-based MIME media types, whatever their particular content may represent. This allows the use of generic XML processors and technologies on a wide variety of different XML document types at a minimum cost, using existing frameworks for media type registration.

Although the use of a suffix was not considered as part of the original MIME architecture, this choice is considered to provide the most functionality with the least potential for interoperability problems or lack of future extensibility. The alternatives to the ' +xml' suffix and the reason for its selection are described in Appendix A.

As XML development continues, new XML document types are appearing rapidly. Many of these XML document types would benefit from the identification possibilities of a more specific MIME media type than text/xml or application/xml can provide, and it is likely that many new media types for XML-based document types will be registered in the near and ongoing future.

While the benefits of specific MIME types for particular types of XML documents are significant, all XML documents share common structures and syntax that make possible common processing.

Some areas where 'generic' processing is useful include:

- Browsing - An XML browser can display any XML document with a provided [CSS] or [XSLT] style sheet, whatever the vocabulary of that document.

- Editing - Any XML editor can read, modify, and save any XML document.

- Fragment identification - XPointers (work in progress) can work with any XML document, whatever vocabulary it uses and whether or not it uses XPointer for its own fragment identification. 
- Hypertext linking - XLink (work in progress) hypertext linking is designed to connect any XML documents, regardless of vocabulary.

- Searching - XML-oriented search engines, web crawlers, agents, and query tools should be able to read XML documents and extract the names and content of elements and attributes even if the tools are ignorant of the particular vocabulary used for elements and attributes.

- Storage - XML-oriented storage systems, which keep XML documents internally in a parsed form, should similarly be able to process, store, and recreate any XML document.

- Well-formedness and validity checking - An XML processor can confirm that any XML document is well-formed and that it is valid (i.e., conforms to its declared DTD or Schema).

When a new media type is introduced for an XML-based format, the name of the media type SHOULD end with '+xml'. This convention will allow applications that can process XML generically to detect that the MIME entity is supposed to be an XML document, verify this assumption by invoking some XML processor, and then process the XML document accordingly. Applications may match for types that represent XML MIME entities by comparing the subtype to the pattern '*/*+xml'. (Of course, 4 of the 5 media types defined in this document -- text/xml, application/xml, text/xml-external-parsed-entity, and application/xml-external-parsed-entity -- also represent XML MIME entities while not conforming to the $' \star / *+x m l$ pattern.)

NOTE: Section 14.1 of HTTP [RFC2616] does not support Accept headers of the form "Accept: */*+xml" and so this header MUST NOT be used in this way. Instead, content negotiation[RFC2703] could potentially be used if an XML-based MIME type were needed.

XML generic processing is not always appropriate for XML-based media types. For example, authors of some such media types may wish that the types remain entirely opaque except to applications that are specifically designed to deal with that media type. By NOT following the naming convention '+xml', such media types can avoid XML-generic processing. Since generic processing will be useful in many cases, however -- including in some situations that are difficult to predict ahead of time -- those registering media types SHOULD use the '+xml' convention unless they have a particularly compelling reason not to.

The registration process for these media types is described in [RFC2048]. The registrar for the IETF tree will encourage new XMLbased media type registrations in the IETF tree to follow this guideline. Registrars for other trees sHould follow this convention 
in order to ensure maximum interoperability of their XML-based documents. Similarly, media subtypes that do not represent XML MIME entities MUST NOT be allowed to register with a '+xml' suffix.

\subsection{Referencing}

Registrations for new XML-based media types under the top-level type "text" SHOULD, in specifying the charset parameter and encoding considerations, define them as: "Same as [charset parameter / encoding considerations] of text/xml as specified in RFC 3023."

Registrations for new XML-based media types under top-level types other than "text" SHOULD, in specifying the charset parameter and encoding considerations, define them as: "Same as [charset parameter / encoding considerations] of application/xml as specified in RFC 3023."

The use of the charset parameter is STRONGLY RECOMMENDED, since this information can be used by XML processors to determine authoritatively the charset of the XML MIME entity.

These registrations SHOULD specify that the XML-based media type being registered has all of the security considerations described in RFC 3023 plus any additional considerations specific to that media type.

These registrations SHOULD also make reference to RFC 3023 in specifying magic numbers, fragment identifiers, base URIs, and use of the BOM.

These registrations MAY reference the text/xml registration in $\mathrm{RFC}$ 3023 in specifying interoperability considerations, if these considerations are not overridden by issues specific to that media type.

8. Examples

The examples below give the value of the MIME Content-type header and the XML declaration (which includes the encoding declaration) inside the XML MIME entity. For UTF-16 examples, the Byte Order Mark character is denoted as " $\{\mathrm{BOM}\}$ ", and the XML declaration is assumed to come at the beginning of the XML MIME entity, immediately following the BOM. Note that other MIME headers may be present, and the XML MIME entity may contain other data in addition to the XML declaration; the examples focus on the Content-type header and the encoding declaration for clarity. 
8.1 Text/xml with UTF-8 Charset

Content-type: text/xml; charset="utf-8"

$<$ ?xml version="1.0" encoding="utf-8"?>

This is the recommended charset value for use with text/xml. Since the charset parameter is provided, MIME and XML processors MUST treat the enclosed entity as UTF-8 encoded.

If sent using a 7-bit transport (e.g., SMTP[RFC0821]), the XML MIME entity MUST use a content-transfer-encoding of either quotedprintable or base64. For an 8-bit clean transport (e.g., 8BITMIME ESMTP or NNTP), or a binary clean transport (e.g., HTTP), no content-transfer-encoding is necessary.

8.2 Text/xml with UTF-16 Charset

Content-type: text/xml; charset="utf-16"

$\{\mathrm{BOM}\}<$ ? xml version=' $1.0^{\prime}$ encoding='utf $-16^{\prime}$ ?>

or

$\{\mathrm{BOM}\}<$ ?xml version='1.0' ?>

This is possible only when the XML MIME entity is transmitted via HTTP, which uses a MIME-like mechanism and is a binary-clean protocol, hence does not perform CR and LF transformations and allows NUL octets. As described in [RFC2781], the UTF-16 family MUST NOT be used with media types under the top-level type "text" except over HTTP (see section 19.4.1 of [RFC2616] for details).

Since HTTP is binary clean, no content-transfer-encoding is necessary.

8.3 Text/xml with UTF-16BE Charset

Content-type: text/xml; charset="utf-16be"

$<$ ?xml version=' $1.0^{\prime}$ encoding=' utf-16be' ?>

Observe that the BOM does not exist. This is again possible only when the XML MIME entity is transmitted via HTTP. 
8.4 Text/xml with ISO-2022-KR Charset

Content-type: text/xml; charset="iso-2022-kr"

<?xml version="1.0" encoding='iso-2022-kr' ?>

This example shows text/xml with a Korean charset (e.g., Hangul) encoded following the specification in [RFC1557]. Since the charset parameter is provided, MIME and XML processors MUST treat the enclosed entity as encoded per RFC 1557.

Since ISO-2022-KR has been defined to use only 7 bits of data, no content-transfer-encoding is necessary with any transport.

8.5 Text/xml with Omitted Charset

Content-type: text/xml

$\{\mathrm{BOM}\}<$ ?xml version="1.0" encoding="utf-16"?>

or

$\{\mathrm{BOM}\}<$ ?xml version="1.0" ?>

This example shows text/xml with the charset parameter omitted. In this case, MIME and XML processors MUST assume the charset is "usascii", the default charset value for text media types specified in [RFC2046]. The default of "us-ascii" holds even if the text/xml entity is transported using HTTP.

Omitting the charset parameter is NOT RECOMMENDED for text/xml. For example, even if the contents of the XML MIME entity are UTF-16 or $\mathrm{UTF}-8$, or the XML MIME entity has an explicit encoding declaration, XML and MIME processors MUST assume the charset is "us-ascii".

8.6 Application/xml with UTF-16 Charset

Content-type: application/xml; charset="utf-16"

$\{\mathrm{BOM}\}<$ ?xml version="1.0" encoding="utf-16"?>

or

$\{\mathrm{BOM}\}<$ ?xml version="1.0" ?>

This is a recommended charset value for use with application/xml. Since the charset parameter is provided, MIME and XML processors MUST treat the enclosed entity as UTF-16 encoded. 
If sent using a 7-bit transport (e.g., SMTP) or an 8-bit clean transport (e.g., 8BITMIME ESMTP or NNTP), the XML MIME entity MUST be encoded in quoted-printable or base64. For a binary clean transport (e.g., HTTP), no content-transfer-encoding is necessary.

8.7 Application/xml with UTF-16BE Charset

Content-type: application/xml; charset="utf-16be"

$<$ ?xml version='1.0' encoding='utf-16be' ?>

Observe that the BOM does not exist. Since the charset parameter is provided, MIME and XML processors MUST treat the enclosed entity as UTF-16BE encoded.

8.8 Application/xml with ISO-2022-KR Charset

Content-type: application/xml; charset="iso-2022-kr"

<?xml version="1.0" encoding="iso-2022-kr"?>

This example shows application/xml with a Korean charset (e.g., Hangul) encoded following the specification in [RFC1557]. Since the charset parameter is provided, MIME and XML processors MUST treat the enclosed entity as encoded per RFC 1557, independent of whether the XML MIME entity has an internal encoding declaration (this example does show such a declaration, which agrees with the charset parameter).

Since ISO-2022-KR has been defined to use only 7 bits of data, no content-transfer-encoding is necessary with any transport.

8.9 Application/xml with Omitted Charset and UTF-16 XML MIME Entity

Content-type: application/xml

$\{\mathrm{BOM}\}<$ ?xml version='1.0' encoding="utf-16"?>

or

$\{\mathrm{BOM}\}<$ ?xml version='1.0' ?>

For this example, the XML MIME entity begins with a BOM. Since the charset has been omitted, a conforming XML processor follows the requirements of [XML], section 4.3.3. Specifically, the XML processor reads the BOM, and thus knows deterministically that the charset is UTF-16. 
An XML-unaware MIME processor SHOULD make no assumptions about the charset of the XML MIME entity.

8.10 Application/xml with Omitted Charset and UTF-8 Entity

Content-type: application/xml

$<$ ?xml version='1.0'?>

In this example, the charset parameter has been omitted, and there is no BOM. Since there is no BOM, the XML processor follows the requirements in section 4.3 .3 of [XML], and optionally applies the mechanism described in Appendix $F$ (which is non-normative) of [XML] to determine the charset encoding of UTF-8. The XML MIME entity does not contain an encoding declaration, but since the encoding is UTF-8, this is still a conforming XML MIME entity.

An XML-unaware MIME processor SHOULD make no assumptions about the charset of the XML MIME entity.

8.11 Application/xml with Omitted Charset and Internal Encoding Declaration

Content-type: application/xml

<?xml version='1.0' encoding="iso-10646-ucs-4"?>

In this example, the charset parameter has been omitted, and there is no BOM. However, the XML MIME entity does have an encoding declaration inside the XML MIME entity that specifies the entity's charset. Following the requirements in section 4.3 .3 of [XML], and optionally applying the mechanism described in Appendix $F$ (nonnormative) of [XML], the XML processor determines the charset of the XML MIME entity (in this example, UCS-4).

An XML-unaware MIME processor SHOULD make no assumptions about the charset of the XML MIME entity.

8.12 Text/xml-external-parsed-entity with UTF-8 Charset

Content-type: text/xml-external-parsed-entity; charset="utf-8"

$<$ ?xml encoding="utf-8"?>

This is the recommended charset value for use with text/xmlexternal-parsed-entity. Since the charset parameter is provided, MIME and XML processors MUST treat the enclosed entity as UTF-8 encoded. 
If sent using a 7-bit transport (e.g., SMTP), the XML MIME entity MUST use a content-transfer-encoding of either quoted-printable or base64. For an 8-bit clean transport (e.g., 8BITMIME ESMTP or NNTP), or a binary clean transport (e.g., HTTP) no content-transfer-encoding is necessary.

8.13 Application/xml-external-parsed-entity with UTF-16 Charset

Content-type: application/xml-external-parsed-entity; charset $=" u t f-16 "$

$\{\mathrm{BOM}\}<$ ?xml encoding="utf-16"?>

or

$\{\mathrm{BOM}\}<$ ? xml ?>

This is a recommended charset value for use with application/xmlexternal-parsed-entity. Since the charset parameter is provided, MIME and XML processors MUST treat the enclosed entity as UTF-16 encoded.

If sent using a 7-bit transport (e.g., SMTP) or an 8-bit clean transport (e.g., 8BITMIME ESMTP or NNTP), the XML MIME entity MUST be encoded in quoted-printable or base64. For a binary clean transport (e.g., HTTP), no content-transfer-encoding is necessary.

8.14 Application/xml-external-parsed-entity with UTF-16BE Charset

Content-type: application/xml-external-parsed-entity; charset $=$ "utf -16 be"

$<$ ?xml encoding="utf-16be"?>

Since the charset parameter is provided, MIME and XML processors MUST treat the enclosed entity as UTF-16BE encoded.

8.15 Application/xml-dtd

Content-type: application/xml-dtd; charset="utf-8"

$<$ ?xml encoding="utf-8"?>

Charset "utf-8" is a recommended charset value for use with application/xml-dtd. Since the charset parameter is provided, MIME and XML processors MUST treat the enclosed entity as UTF-8 encoded. 


\subsection{Application/mathml+xml}

Content-type: application/mathml+xml

$<$ ?xml version="1.0" ?>

MathML documents are XML documents whose content describes mathematical information, as defined by [MathML]. As a format based on XML, MathML documents SHOULD use the '+xml' suffix convention in their MIME content-type identifier. However, no content type has yet been registered for MathML and so this media type should not be used until such registration has been completed.

8.17 Application/xslt $+x m l$

Content-type: application/xslt+xml

$<$ ?xml version="1.0" ?>

Extensible Stylesheet Language (XSLT) documents are XML documents whose content describes stylesheets for other XML documents, as defined by [XSLT]. As a format based on XML, XSLT documents SHOULD use the ' $+x m^{\prime}$ ' suffix convention in their MIME content-type identifier. However, no content type has yet been registered for XSLT and so this media type should not be used until such registration has been completed.

\subsection{Application/rdf $+x m l$}

Content-type: application/rdf+xml

$<$ ?xml version="1.0" ?>

RDF documents identified using this MIME type are XML documents whose content describes metadata, as defined by [RDF]. As a format based on XML, RDF documents SHOULD use the '+xml' suffix convention in their MIME content-type identifier. However, no content type has yet been registered for $\mathrm{RDF}$ and so this media type should not be used until such registration has been completed.

\subsection{Image/svg+xml}

Content-type: image/svg+xml

<?xml version="1.0" ?> 
Scalable Vector Graphics (SVG) documents are XML documents whose content describes graphical information, as defined by [SVG]. As a format based on XML, SVG documents SHOULD use the '+xml' suffix convention in their MIME content-type identifier. However, no content type has yet been registered for SVG and so this media type should not be used until such registration has been completed.

\subsection{INCONSISTENT EXAMPLE: Text/xml with UTF-8 Charset}

Content-type: text/xml; charset="utf-8"

$<$ ?xml version="1.0" encoding="iso-8859-1"?>

Since the charset parameter is provided in the Content-Type header, MIME and XML processors MUST treat the enclosed entity as UTF-8 encoded. That is, the "iso-8859-1" encoding MUST be ignored.

Processors generating XML MIME entities MUST NOT label conflicting charset information between the MIME Content-Type and the XML declaration.

\section{IANA Considerations}

As described in section 7, this document updates the [RFC2048] registration process for XML-based MIME types.

10. Security Considerations

XML, as a subset of SGML, has all of the same security considerations as specified in [RFC1874], and likely more, due to its expected ubiquitous deployment.

To paraphrase section 3 of RFC 1874, XML MIME entities contain information to be parsed and processed by the recipient's XML system. These entities may contain and such systems may permit explicit system level commands to be executed while processing the data. To the extent that an XML system will execute arbitrary command strings, recipients of XML MIME entities may be a risk. In general, it may be possible to specify commands that perform unauthorized file operations or make changes to the display processor's environment that affect subsequent operations.

In general, any information stored outside of the direct control of the user -- including CSS style sheets, XSL transformations, entity declarations, and DTDs -- can be a source of insecurity, by either obvious or subtle means. For example, a tiny "whiteout attack" modification made to a "master" style sheet could make words in critical locations disappear in user documents, without directly 
modifying the user document or the stylesheet it references. Thus, the security of any XML document is vitally dependent on all of the documents recursively referenced by that document.

The entity lists and DTDs for XHTML 1.0[XHTML], for instance, are likely to be a commonly used set of information. Many developers will use and trust them, few of whom will know much about the level of security on the W3C's servers, or on any similarly trusted repository.

The simplest attack involves adding declarations that break validation. Adding extraneous declarations to a list of character entities can effectively "break the contract" used by documents. A tiny change that produces a fatal error in a DTD could halt XML processing on a large scale. Extraneous declarations are fairly obvious, but more sophisticated tricks, like changing attributes from being optional to required, can be difficult to track down. Perhaps the most dangerous option available to crackers is redefining default values for attributes: e.g., if developers have relied on defaulted attributes for security, a relatively small change might expose enormous quantities of information.

Apart from the structural possibilities, another option, "entity spoofing," can be used to insert text into documents, vandalizing and perhaps conveying an unintended message. Because XML 1.0 permits multiple entity declarations, and the first declaration takes precedence, it's possible to insert malicious content where an entity is used, such as by inserting the full text of Winnie the Pooh in every occurrence of \&mdash; .

Use of the digital signatures work currently underway by the xmldsig working group may eventually ameliorate the dangers of referencing external documents not under one's own control.

Use of XML is expected to be varied, and widespread. XML is under scrutiny by a wide range of communities for use as a common syntax for community-specific metadata. For example, the Dublin Core[RFC2413] group is using XML for document metadata, and a new effort has begun that is considering use of XML for medical information. Other groups view XML as a mechanism for marshalling parameters for remote procedure calls. More uses of XML will undoubtedly arise.

Security considerations will vary by domain of use. For example, XML medical records will have much more stringent privacy and security considerations than XML library metadata. Similarly, use of XML as a parameter marshalling syntax necessitates a case by case security review. 
XML may also have some of the same security concerns as plain text. Like plain text, XML can contain escape sequences that, when displayed, have the potential to change the display processor environment in ways that adversely affect subsequent operations. Possible effects include, but are not limited to, locking the keyboard, changing display parameters so subsequent displayed text is unreadable, or even changing display parameters to deliberately obscure or distort subsequent displayed material so that its meaning is lost or altered. Display processors SHould either filter such material from displayed text or else make sure to reset all important settings after a given display operation is complete.

Some terminal devices have keys whose output, when pressed, can be changed by sending the display processor a character sequence. If this is possible the display of a text object containing such character sequences could reprogram keys to perform some illicit or dangerous action when the key is subsequently pressed by the user. In some cases not only can keys be programmed, they can be triggered remotely, making it possible for a text display operation to directly perform some unwanted action. As such, the ability to program keys SHOULD be blocked either by filtering or by disabling the ability to program keys entirely.

Note that it is also possible to construct XML documents that make use of what XML terms "entity references" (using the XML meaning of the term "entity" as described in Section 2), to construct repeated expansions of text. Recursive expansions are prohibited by [XML] and XML processors are required to detect them. However, even nonrecursive expansions may cause problems with the finite computing resources of computers, if they are performed many times.

References

[ASCII] "US-ASCII. Coded Character set -- 7-Bit American Standard Code for Information Interchange", ANSI X3.4-1986, 1986.

[CSS] Bos, B., Lie, H.W., Lilley, C. and I. Jacobs, "Cascading Style Sheets, level 2 (CSS2) Specification", World Wide Web Consortium Recommendation REC-CSS2, May 1998, <http: //www.w3.org/TR/REC-CSS2/>.

[ISO8859] "ISO-8859. International Standard -- Information Processing -- 8-bit Single-Byte Coded Graphic Character Sets -- Part 1: Latin alphabet No. 1, ISO-8859-1:1987", 1987 . 
[MathML] Ion, P. and R. Miner, "Mathematical Markup Language (MathML) 1.01", World Wide Web Consortium Recommendation REC-MathML, July 1999, <http://www.w3.org/TR/REC-MathML/>.

[PNG] Boutell, T., "PNG (Portable Network Graphics) Specification", World Wide Web Consortium Recommendation REC-png, October 1996, <http://www.w3.org/TR/REC-png>.

[RDF] Lassila, O. and R.R. Swick, "Resource Description Framework (RDF) Model and Syntax Specification", World Wide Web Consortium Recommendation REC-rdf-syntax, February 1999, <http://www.w3.org/TR/REC-rdf-syntax/>.

[RFC0821] Postel, J., "Simple Mail Transfer Protocol", STD 10, RFC 821, August 1982 .

[RFC0977] Kantor, B. and P. Lapsley, "Network News Transfer Protocol", RFC 977, February 1986.

[RFC1557] Choi, U., Chon, K. and H. Park, "Korean Character Encoding for Internet Messages", RFC 1557, December 1993.

[RFC1652] Klensin, J., Freed, N., Rose, M., Stefferud, E. and D. Crocker, "SMTP Service Extension for 8bit-MIMEtransport", RFC 1652, July 1994 .

[RFC1874] Levinson, E., "SGML Media Types", RFC 1874, December 1995.

[RFC2045] Freed, N. and N. Borenstein, "Multipurpose Internet Mail Extensions (MIME) Part One: Format of Internet Message Bodies", RFC 2045, November 1996.

[RFC2046] Freed, N. and N. Borenstein, "Multipurpose Internet Mail Extensions (MIME) Part Two: Media Types", RFC 2046, November 1996.

[RFC2048] Freed, N., Klensin, J. and J. Postel, "Multipurpose Internet Mail Extensions (MIME) Part Four: Registration Procedures", RFC 2048, November 1996.

[RFC2060] Crispin, M., "Internet Message Access Protocol - Version 4 rev1", RFC 2060, December 1996.

[RFC2077] Nelson, S., Parks, C. and Mitra, "The Model Primary Content Type for Multipurpose Internet Mail Extensions", RFC 2077, January 1997. 
[RFC2119] Bradner, S., "Key words for use in RFCs to Indicate Requirement Levels", BCP 14, RFC 2119, March 1997.

[RFC2130] Weider, C., Preston, C., Simonsen, K., Alvestrand, H., Atkinson, R., Crispin, M. and P. Svanberg, "The Report of the IAB Character Set Workshop held 29 February - 1 March, 1996", RFC 2130, April 1997.

[RFC2279] Yergeau, F., "UTF-8, a transformation format of ISO $10646 "$, RFC 2279, January 1998.

[RFC2376] Whitehead, E. and M. Murata, "XML Media Types", RFC 2376, July 1998 .

[RFC2396] Berners-Lee, T., Fielding, R. and L. Masinter, "Uniform Resource Identifiers (URI): Generic Syntax.", RFC 2396, August 1998 .

[RFC2413] Weibel, S., Kunze, J., Lagoze, C. and M. Wolf, "Dublin Core Metadata for Resource Discovery", RFC 2413, September 1998 .

[RFC2445] Dawson, F. and D. Stenerson, "Internet Calendaring and Scheduling Core Object Specification (iCalendar)", RFC 2445, November 1998 .

[RFC2518] Goland, Y., Whitehead, E., Faizi, A., Carter, S. and D. Jensen, "HTTP Extensions for Distributed Authoring -WEBDAV", RFC 2518, February 1999.

[RFC2616] Fielding, R., Gettys, J., Mogul, J., Nielsen, H., Masinter, L., Leach, P. and T. Berners-Lee, "Hypertext Transfer Protocol -- HTTP/1.1", RFC 2616, June 1999.

[RFC2629] Rose, M., "Writing I-Ds and RFCs using XML", RFC 2629, June 1999.

[RFC2703] Klyne, G., "Protocol-independent Content Negotiation Framework", RFC 2703, September 1999.

[RFC2781] Hoffman, P. and F. Yergeau, "UTF-16, an encoding of ISO 10646", RFC 2781, Februrary 2000 .

[RFC2801] Burdett, D., "Internet Open Trading Protocol - IOTP Version 1.0", RFC 2801, April 2000. 
[SGML] International Standard Organization, "Information Processing -- Text and Office Systems -- Standard Generalized Markup Language (SGML)", ISO 8879, October 1986 .

[SVG] Ferraiolo, J., "Scalable Vector Graphics (SVG) ", World Wide Web Consortium Candidate Recommendation SVG, November 2000, <http://www.w3.org/TR/SVG>.

[XHTML] Pemberton, S. and et al, "XHTML 1.0: The Extensible HyperText Markup Language", World Wide Web Consortium Recommendation xhtml1, January 2000, <http://www.w3.org/TR/xhtml1>.

[XML] Bray, T., Paoli, J., Sperberg-McQueen, C.M. and E. Maler, "Extensible Markup Language (XML) 1.0 (Second Edition)", World Wide Web Consortium Recommendation REC-xml, October 2000, <http://Www.w3.org/TR/REC-xml>.

[XSLT] Clark, J., "XSL Transformations (XSLT) Version 1.0", World Wide Web Consortium Recommendation xslt, November 1999, <http://wWw.w3.org/TR/xslt>. 


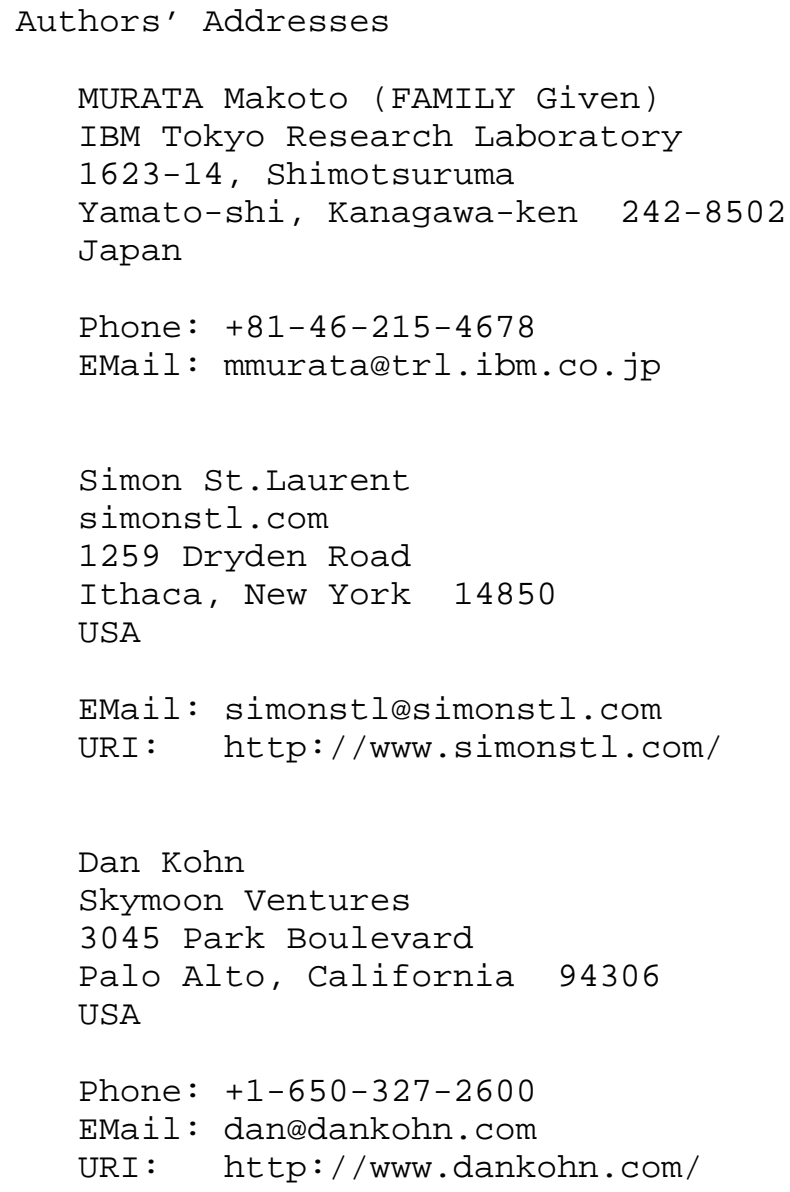


Appendix A. Why Use the '+xml' Suffix for XML-Based MIME Types?

Although the use of a suffix was not considered as part of the original MIME architecture, this choice is considered to provide the most functionality with the least potential for interoperability problems or lack of future extensibility. The alternatives to the ' $+\mathrm{xml}^{\prime}$ suffix and the reason for its selection are described below.

A.1 Why not just use text/xml or application/xml and let the XML processor dispatch to the correct application based on the referenced DTD?

text/xml and application/xml remain useful in many situations, especially for document-oriented applications that involve combining XML with a stylesheet in order to present the data. However, XML is also used to define entirely new data types, and an XML-based format such as image/svg+xml fits the definition of a MIME media type exactly as well as image/png[PNG] does. (Note that image/svg+xml is not yet registered.) Although extra functionality is available for MIME processors that are also XML processors, XML-based media types -- even when treated as opaque, non-XML media types -- are just as useful as any other media type and should be treated as such.

Since MIME dispatchers work off of the MIME type, use of text/xml or application/xml to label discrete media types will hinder correct dispatching and general interoperability. Finally, many XML documents use neither DTDs nor namespaces, yet are perfectly legal XML .

A.2 Why not create a new subtree (e.g., image/xml.svg) to represent XML MIME types?

The subtree under which a media type is registered -- IETF, vendor (*/vnd.*), or personal (*/prs.*); see [RFC2048] for details -- is completely orthogonal from whether the media type uses XML syntax or not. The suffix approach allows XML document types to be identified within any subtree. The vendor subtree, for example, is likely to include a large number of XML-based document types. By using a suffix, rather than setting up a separate subtree, those types may remain in the same location in the tree of MIME types that they would have occupied had they not been based on XML.

A.3 Why not create a new top-level MIME type for XML-based media types?

The top-level MIME type (e.g., model/*[RFC2077]) determines what kind of content the type is, not what syntax it uses. For example, agents using image/* to signal acceptance of any image format should certainly be given access to media type image/svg+xml, which is in 
all respects a standard image subtype. It just happens to use XML to describe its syntax. The two aspects of the media type are completely orthogonal.

XML-based data types will most likely be registered in ALL top-level categories. Potential, though currently unregistered, examples could include application/mathml+xml[MathML] and image/svg+xml[SVG] .

A.4 Why not just have the MIME processor 'sniff' the content to determine whether it is XML?

Rather than explicitly labeling XML-based media types, the processor could look inside each type and see whether or not it is XML. The processor could also cache a list of XML-based media types.

Although this method might work acceptably for some mail applications, it would fail completely in many other uses of MIME. For instance, an XML-based web crawler would have no way of determining whether a file is XML except to fetch it and check. The same issue applies in some IMAP4[RFC2060] mail applications, where the client first fetches the MIME type as part of the message structure and then decides whether to fetch the MIME entity. Requiring these fetches just to determine whether the MIME type is XML could have significant bandwidth and latency disadvantages in many situations.

Sniffing XML also isn't as simple as it might seem. DOCTYPE declarations aren't required, and they can appear fairly deep into a document under certain unpreventable circumstances. (E.g., the XML declaration, comments, and processing instructions can occupy space before the DOCTYPE declaration.) Even sniffing the DOCTYPE isn't completely reliable, thanks to a variety of issues involving default values for namespaces within external DTDs and overrides inside the internal DTD. Finally, the variety in potential character encodings (something XML provides tools to deal with), also makes reliable sniffing less likely.

A.5 Why not use a MIME parameter to specify that a media type uses XML syntax?

For example, one could use "Content-Type: application/iotp; alternate-type=text/xml" or "Content-Type: application/iotp; syntax=xml" .

Section 5 of [RFC2045] says that "Parameters are modifiers of the media subtype, and as such do not fundamentally affect the nature of the content". However, all XML-based media types are by their nature 
always XML. Parameters, as they have been defined in the MIME architecture, are never invariant across all instantiations of a media type.

More practically, very few if any MIME dispatchers and other MIME agents support dispatching off of a parameter. While MIME agents on the receiving side will need to be updated in either case to support (or fall back to) generic XML processing, it has been suggested that it is easier to implement this functionality when acting off of the media type rather than a parameter. More important, sending agents require no update to properly tag an image as "image/svg+xml", but few if any sending agents currently support always tagging certain content types with a parameter.

A. 6 How about labeling with parameters in the other direction (e.g., application/xml; Content-Feature=iotp)?

This proposal fails under the simplest case, of a user with neither knowledge of XML nor an XML-capable MIME dispatcher. In that case, the user's MIME dispatcher is likely to dispatch the content to an XML processing application when the correct default behavior should be to dispatch the content to the application responsible for the content type (e.g., an ecommerce engine for application/iotp+xml[RFC2801], once this media type is registered).

Note that even if the user had already installed the appropriate application (e.g., the ecommerce engine), and that installation had updated the MIME registry, many operating system level MIME registries such as . mailcap in Unix and HKEY_CLASSES_ROOT in Windows do not currently support dispatching off a parameter, and cannot easily be upgraded to do so. And, even if the operating system were upgraded to support this, each MIME dispatcher would also separately need to be upgraded.

A. 7 How about a new superclass MIME parameter that is defined to apply to all MIME types (e.g., Content-Type: application/iotp; \$superclass $=\mathrm{xml})$ ?

This combines the problems of Appendix A.5 and Appendix A. 6 .

If the sender attaches an image/svg+xml file to a message and includes the instructions "Please copy the French text on the road sign", someone with an XML-aware MIME client and an XML browser but no support for SVG can still probably open the file and copy the text. By contrast, with superclasses, the sender must add superclass support to her existing mailer AND the receiver must add superclass support to his before this transaction can work correctly. 
If the receiver comes to rely on the superclass tag being present and applications are deployed relying on that tag (as always seems to happen), then only upgraded senders will be able to interoperate with those receiving applications.

A.8 What about adding a new parameter to the Content-Disposition header or creating a new content-Structure header to indicate XML syntax?

This has nearly identical problems to Appendix A.7, in that it requires both senders and receivers to be upgraded, and few if any operating systems and MIME dispatchers support working off of anything other than the MIME type.

A. 9 How about a new Alternative-Content-Type header?

This is better than Appendix A.8, in that no extra functionality needs to be added to a MIME registry to support dispatching of information other than standard content types. However, it still requires both sender and receiver to be upgraded, and it will also fail in many cases (e.g., web hosting to an outsourced server), where the user can set MIME types (often through implicit mapping to file extensions), but has no way of adding arbitrary HTTP headers.

A.10 How about using a conneg tag instead (e.g., accept-features: $($ syntax $=x m l))$ ?

When the conneg protocol is fully defined, this may potentially be a reasonable thing to do. But given the limited current state of conneg[RFC2703] development, it is not a credible replacement for a MIME-based solution .

A.11 How about a third-level content-type, such as text/xml/rdf?

MIME explicitly defines two levels of content type, the top-level for the kind of content and the second-level for the specific media type. [RFC2048] extends this in an interoperable way by using prefixes to specify separate trees for IETF, vendor, and personal registrations. This specification also extends the two-level type by using the ' +xml' suffix. In both cases, processors that are unaware of these later specifications treat them as opaque and continue to interoperate. By contrast, adding a third-level type would break the current MIME architecture and cause numerous interoperability failures. 
A.12 Why use the plus $\left({ }^{\prime}+{ }^{\prime}\right)$ character for the suffix '+xml'?

As specified in section 5.1 of [RFC2045], a tspecial can't be used:

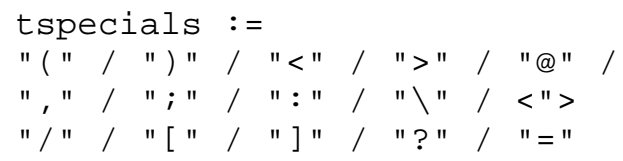

It was thought that "." would not be a good choice since it is already used as an additional hierarchy delimiter. Also, "*" has a common wildcard meaning, and "-" and "-" are common word separators and easily confused. The characters \%' '\#\& are frequently used for quoting or comments and so are not ideal.

That leaves: $\sim ! \$^{\wedge}+\{\} \mid$

Note that "-" is used heavily in the current registry. "\$" and "_" are used once each. The others are currently unused.

It was thought that ' + ' expressed the semantics that a MIME type can be treated (for example) as both scalable vector graphics AND ALSO as XML; it is both simultaneously.

A.13 What is the semantic difference between application/foo and application/foo+xml?

MIME processors that are unaware of XML will treat the '+xml' suffix as completely opaque, so it is essential that no extra semantics be assigned to its presence. Therefore, application/foo and application/foo+xml SHOULD be treated as completely independent media types. Although, for example, text/calendartxml could be an XML version of text/calendar[RFC2445], it is possible that this (hypothetical) new media type would include new semantics as well as new syntax, and in any case, there would be many applications that support text/calendar but had not yet been upgraded to support text/calendar+xml.

A.14 What happens when an even better markup language (e.g., EBML) is defined, or a new category of data?

In the ten years that MIME has existed, XML is the first generic data format that has seemed to justify special treatment, so it is hoped that no further suffixes will be necessary. However, if some are later defined, and these documents were also XML, they would need to specify that the 'txml' suffix is always the outermost suffix (e.g., 
application/footebml+xml not application/foo+xml+ebml). If they were not XML, then they would use a regular suffix (e.g., application/foo+ebml).

A.15 Why must I use the '+xml' suffix for my new XML-based media type?

You don't have to, but unless you have a good reason to explicitly disallow generic XML processing, you should use the suffix so as not to curtail the options of future users and developers.

Whether the inventors of a media type, today, design it for dispatch to generic XML processing machinery (and most won't) is not the critical issue. The core notion is that the knowledge that some media type happens to use XML syntax opens the door to unanticipated kinds of processing beyond those envisioned by its inventors, and on this basis identifying such encoding is a good and useful thing.

Developers of new media types are often tightly focused on a particular type of processing that meets current needs. But there is no need to rule out generic processing as well, which could make your media type more valuable over time. It is believed that registering with the '+xml' suffix will cause no interoperability problems whatsoever, while it may enable significant new functionality and interoperability now and in the future. So, the conservative approach is to include the 'txml' suffix.

Appendix B. Changes from RFC 2376

There are numerous and significant differences between this specification and [RFC2376], which it obsoletes. This appendix summarizes the major differences only.

First, text/xml-external-parsed-entity and application/xml-externalparsed-entity are added as media types for external parsed entities, and text/xml and application/xml are now prohibited.

Second, application/xml-dtd is added as a media type for external DTD subsets and external parameter entities, and text/xml and application/xml are now prohibited.

Third, "utf-16le" and "utf-16be" are added. RFC 2781 has introduced these BOM-less variations of the UTF-16 family.

Fourth, a naming convention ('+xml') for XML-based media types has been added, which also updates [RFC2048] as described in section 7 . By following this convention, an XML-based media type can be easily recognized as such. 
Appendix C. Acknowledgements

This document reflects the input of numerous participants to the ietf-xml-mimedimc.org mailing list, though any errors are the responsibility of the authors. Special thanks to:

Mark Baker, James Clark, Dan Connolly, Martin Duerst, Ned Freed, Yaron Goland, Rick Jelliffe, Larry Masinter, David Megginson, Keith Moore, Chris Newman, Gavin Nicol, Marshall Rose, Jim Whitehead and participants of the XML activity at the $\mathrm{W} 3 \mathrm{C}$. 
Full Copyright statement

Copyright (C) The Internet Society (2001). All Rights Reserved.

This document and translations of it may be copied and furnished to others, and derivative works that comment on or otherwise explain it or assist in its implementation may be prepared, copied, published and distributed, in whole or in part, without restriction of any kind, provided that the above copyright notice and this paragraph are included on all such copies and derivative works. However, this document itself may not be modified in any way, such as by removing the copyright notice or references to the Internet society or other Internet organizations, except as needed for the purpose of developing Internet standards in which case the procedures for copyrights defined in the Internet Standards process must be followed, or as required to translate it into languages other than English.

The limited permissions granted above are perpetual and will not be revoked by the Internet society or its successors or assigns.

This document and the information contained herein is provided on an "AS IS" basis and THE INTERNET SOCIETY AND THE INTERNET ENGINEERING TASK FORCE DISCLAIMS ALL WARRANTIES, EXPRESS OR IMPLIED, INCLUDING BUT NOT LIMITED TO ANY WARRANTY THAT THE USE OF THE INFORMATION HEREIN WILL NOT INFRINGE ANY RIGHTS OR ANY IMPLIED WARRANTIES OF MERCHANTABILITY OR FITNESS FOR A PARTICULAR PURPOSE.

Acknowledgement

Funding for the RFC Editor function is currently provided by the Internet society. 